\title{
Die Scham der Gestalter
}

\section{Chance für eine überfällige Intervention}

\section{MARTin SAMBAUER}

Positioning of an advertising and television designer. Unleashed by ecological and social destruction, a vague sense of shame is spreading amongst the players of business life. This shame can become a performance driver for a new rationality of responsibility - for intervention in our overheated system. Goverments' discourse becomes the ideal business relationship amongst all economic subjects.

Keywords: Verantwortungsrationalität, Diskursdefizit, Spaltung des gesellschaftlichen Handlungsraumes, der dissoziierte Mensch, neue (Wirtschafts-) Anthropologie.

„Ein Schiff führen (gouverner)“ (Foucault 2004: 146) gilt in der Philosophiegeschichte als beinahe „unvermeidliche Metapher“ (ebd.) ${ }^{1}$ für das Regieren. Michel Foucault verweist 1978 in seiner wegweisenden Vorlesung über die Kunst des Regierens auf die „Beziehung zwischen den Seeleuten und dem Schiff, das gerettet werden, und der Ladung, die in den Hafen gebracht werden muß“ (ebd.). Er charakterisiert diese traditionsreiche Auffassung gegenüber dem Verantwortungsgeflecht von Regierenden zu Regierten durch einen weiteren Vergleich:

„Eine Familie zu führen hat im Grunde genommen nicht hauptsächlich die Rettung des Familieneigentums zum Ziel, sondern Zielobjekt, Zielscheibe sind im Wesentlichen die Individuen, welche die Familie, ihren Reichtum und ihren Wohlstand bilden." (ebd.: 147)

Wer den Begriff corporate governance in der Diskussion um eine gute Unternehmensführung verwendet, bezieht sich bewusst oder unbewusst auf eine begriffsgeschichtliche Tradition, die an vielen Stellen Dissonanzen zur unternehmerischen Praxis der Gegenwart aufweist. Ein Schiffskapitän, der bei einem drohenden Sturm die schwächeren Teile der Mannschaft über Bord werfen lässt, entspricht nur in einem dramatischen Extremfall dem oben skizzierten Ethos des Regierens, durchaus aber einem gängigen Verhaltensmuster heutiger Manager. Ist es vor dem Hintergrund derartiger begrifflicher Dissonanzen überhaupt sinnvoll, die klassische Denktradition des Regierens in der Diskussion um die corporate governance zu berücksichtigen? Falls nicht, warum wird

Martin Sambauer (Regisseur), Das Integral - Büro für Inszenierung und Kommunikation GmbH, Hessstraße 79, D-80796 München, Tel.: +49-(0)89-5527974-0, Fax: +49-(0)89-5527974-15, E-Mail: martin.sambauer@dasintegral.eu.

$1 \quad$ Hinweis bei Foucault (2004: 170) zum klassischen Gebrauch dieser Metapher bei Platon, Eutyphron, 14 b, Protagoras, 325 c, Politeia, 389 d, 488 a - 489 d, 551 c, 573 d, Politikos (Der Staatsmann), 296 e - 297 a, 301 d, 302 a, 304 a, Nomoi, 737 a, 942 b, 945 c, 961 c, usw.; (vgl. P. Louis, Les métaphores de Platon, Paris (Les Belles Lettres) 1945, S. 156; Aristoteles, Poliktik, III, 4, 1276 b, 20 -30; Cicero, Ad Atticum, 10, 8, 6; De republica, 3, 47; Thomas von Aquin, De regno (/De regiminie principum), I, 2. 
der Begriff governance dann eingeführt? Geht es nur um eine schmeichelhafte Charakterisierung des Verfügungsanspruchs, der Machtfülle der Führenden oder sollen doch Ansätze einer vitalpolitischen (vgl. Ulrich 2001: 339ff.) oder sonst wie weiter greifenden Verantwortung ins unternehmerische Denken eingeführt werden?

Regieren bedeutet in dem eingangs beschriebenen Beispiel, eine differenzierte Rationalität der Verantwortung gegenüber den Regierten und dem unterstellten Hoheitsraum herauszubilden ${ }^{2}$ und das Regierungshandeln danach auszurichten. Unsere Gesellschaften sind komplexe Gebilde aus ineinander verschachtelten juridischen Hoheitsräumen, von denen besonders die Unternehmen von einem Regieren, von einer Rationalität der $V$ erantwortung oftmals noch weit entfernt sind. Sie werden nicht regiert, sondern innerhalb der Grenzen einer normativen ökonomischen Umgebungslogik verwaltet. Der Begriff des Regierens kann dagegen neue Aspekte von Autonomie und Verantwortung im Verhältnis der Wirtschaftssubjekte zueinander aufwerfen, die geeignet sind, insgesamt zu einem zukunftsfähigen Wirtschaften zu kommen.

Wenn wir zugestehen, dass jedem Menschen, selbst dem machtlosesten, zumindest sein begrenzter privater Hoheitsraum unwiderruflich unterstellt ist und er dessen Rationalität der Verantwortung ähnlich einem Regierungshandeln verantwortet, dann erweitert sich der sonst eher elitär gedachte Kreis der Regierenden auf alle. In Abhängigkeit von sozialer Stellung oder beruflicher Befugnis dehnt sich der Hoheitsraum des jeweiligen Subjektes gegebenenfalls weiter aus, bis hin zu den weiträumigen Verfügungsbereichen von Staats- und Unternehmenslenkern. Häufig überschneiden sich die Hoheitsräume verschiedener Subjekte, was in dieser Denkweise aber selbst bei hierarchischen Abhängigkeitsverhältnissen keinen der Akteure aus seiner Verantwortung entlässt. Denn das doppelt oder mehrfach betroffene Verfügungsobjekt bleibt in jedem Fall Reflexionsobjekt der eigenen Verantwortungsrationalität. Es befindet sich ja innerhalb des eigenen Hoheitsraumes. Jede Handlung wird zu einem hoheitlichen Akt, dessen Logik und Auswirkungen sich in einem vernunftbildenden Prozess widerspiegeln müssen.

Eine solche multiple Verortung von Verantwortungsrationalität widerspricht der ethischen „Topologie“ (vgl. Ulrich 2001: 289ff.) der Moralökonomik, die den „systematischen Ort der Moral" (vgl. Homann/Blome-Drees 1992: 35) in die Rahmenordnung verweist und gegen eine „Remoralisierung“ (vgl. Homann 1994: 44) 3 des Marktes plädiert. Auch wenn der Begriff der Verantwortungsrationalität in seinem teils kongruenten, teils widersprüchlichen, teils abhängigen, teils differenzierenden, teils normativen Verhältnis zur Moral längst nicht geklärt ist, dürfte zumindest klar sein, dass die Moralökonomik mit der weitgehenden Entlassung des Subjekts aus der moralischen Reflexion seiner Spielzüge auch die Herausbildung einer Verantwortungs-

Etzioni (1994) beschreibt die kognitive Arbeit, die notwendig ist, um zu einer Rationalität zu kommen. Ich vermute, die Ressourcen (Zeit, Recherche, Informationen, Geld, Ort, Teilnehmer), die von Unternehmen erklärtermaßen zur Herausbildung einer Verantwortungsrationalität investiert werden, könnten mittels ökonomischer Bilanzierungstechnik Bemessungsgrundlage für eine Diskussion um Verantwortungsrationalität werden.

$3 \quad$ Karl Homann (1994: 44, 33) wird von Peter Ulrich zitiert:,,Eine Re-Moralisierung der Wirtschaft verbietet sich', denn es sei, jede Re-Moralisierung von Handeln in ausdifferenzierten Teilsystemen nicht nur störend, sie führt sogar zu einer Erosion der Moral (...).““ (Ulrich 2001: 166) 
rationalität annulliert. Ich vermute in diesem Bereich einen Fehlschluss, da man die Moral konsequent der Rationalität des staatlichen Regierungsapparates und der durch sie gebildeten Rahmenordnung überantwortet, dabei aber übersieht, dass genau diese Rationalität der Regierenden von den Erfindern des freien Marktes als derart begrenzt eingestuft wurde, dass sie sich veranlasst sahen, die Versorgung der Bevölkerung mit Gütern lieber den Kräften der Selbstorganisation ${ }^{4}$ zu überlassen als der Unvernunft der Regierung. Die Verteilung der Ressourcen wird nach dieser Logik also der tendenziell unbegrenzten Vernunft des freien Marktes überantwortet, die Moral aber der eindeutig begrenzten Vernunft der Regierung und der durch sie produzierten Rahmenordnung. Dass es in diesem ungleichen Verhältnis zu ethischen, ökologischen oder sozialen Schieflagen kommen wird, liegt nahe. Die verschiedenen Geschwindigkeiten, mit denen der freie Markt einerseits ständig völlig neuartige ethische Problemstellungen hervorbringt und andererseits die demokratisch-träge Rahmenordnung nur verzögert darauf reagieren kann, bilden nur einen von vielen problemerzeugenden Mechanismen dieses Denkens. Nun können wir in Anerkenntnis dieses möglichen Konstruktionsfehlers natürlich nicht in den „Paläoliberalismus“(Ulrich 2001: 340ff.) zurückfallen und uns der vielfach gescheiterten Binnenmoral der unsichtbaren Hand überantworten. Vielmehr sollten durch die Wirtschaftsethik und in der Folge durch die Institutionen aktiv die Verantwortungsverantwortung eines jeden Regierungshandelns, wie auch die hoheitlichen Befugnisse und Pflichten eines jeden Subjektes benannt, gefördert und zugestanden werden, wodurch jeder Handelnde für jede seiner Handlungen beginnt, in Verantwortung zu stehen. Die deliberative Rückfrage wird mit zunehmender Anerkenntnis dieser Haltung zum vernunftbildenden Kommunikationsprinzip im Diskurs der Regierungen, wie ich das ideale Geschäftsverhältnis aller Wirtschaftssubjekte zueinander nennen möchte.

Dieser Ansatz ist mit Sicherheit inspiriert von meiner Fassungslosigkeit gegenüber der Verantwortungsnegation, die wir heute in jedem Supermarkt antreffen. Dort finden sich beispielsweise Frühstücksflocken von renommierten Herstellern, die gezielt in großen Lettern „mit Honig und Nüssen“ der bildungsfernen Mutter die physiologische Unbedenklichkeit des Produktes suggerieren, während der aufgeklärte Beobachter im Kleingedruckten erkennen kann, dass der Gewichtsanteil des Zuckers 33\% beträgt. Um die Unbedenklichkeitssuggestion zu komplettieren, stehen unter diesem Zuckeranteil aber nicht 33\%, sondern vielmehr 12\%, was sich bei genauem Hinsehen auf den Tagesbedarf eines durchschnittlichen Erwachsenen pro 100g Flocken bezieht. Wenn man gleichzeitig den rasant wachsenden Anteil an Diabetikern betrachtet, dann fragt man sich, wie die Werbeleiter, Produktmanager und selbst Grafiker, die an diesem Produkt und seiner Kommunikation beteiligt sind, ruhigen Gewissens ihre hoffentlich wohlbehüteten Kinder ins Bett und zur Schule bringen können, vermutlich ahnend, was sie da in einer anonymen Ferne an den Kindern ihrer bildungsdeprivierten Zeitgenossen anrichten. Ich vermute die Antwort im gesellschaftlichen Sedi-

$4 \quad$ Foucault (2004: 47) zitiert bzgl. der Idee des Laissez-Faire René-Louis de Voyer, Marquis d‘Argenson (1694-1757), Staatssekretär für Auslandsangelegenheiten von 1744-1747: „Man erzählt, daß Michel Colbert mehrere Handelsvertreter bei sich versammelte, um sie zu fragen, was er für den Handel tun könnte; der vernünftigste und am wenigsten schmeichlerische unter ihnen sagte ihm diesen einen Satz: Lassen Sie uns nur machen. Hat man jemals genügend über den tiefen Sinn dieses Satzes nachgedacht? Das ist nur der Versuch eines Kommentars." 
mentieren solcher Formulierungen: „Die Effizienz in den Spielzügen, die Moral in den Spielregeln“ (Homann/Blome-Drees 1992: 35).

Da es keine Spielregeln gibt, die den beschriebenen Vorgang sozialkompatibel regeln, und aufgrund des oben beschriebenen Konstruktionsfehlers stets neue derartige Probleme auftreten werden, stellt sich hinsichtlich des verantwortungsrationalen Ansatzes die folgende Frage: Warum oder unter welchen Bedingungen soll die Gestaltung der Frühstücksflocken-Packung als Verfügungsobjekt eines Werbeleiters zum Reflexionsgegenstand seiner Verantwortungsrationalität werden, wenn sich ihre problematischen Auswirkungen weder innerhalb seines Hoheitsraumes vollziehen, noch auf den ersten Blick direkte Rückwirkungen abzusehen sind? Ich denke, dass die Bereitschaft zur Verantwortungsübernahme eine jener anthropologischen Konstanten ist, aus der heraus sich Gesellschaften konstituieren. ${ }^{5}$ So gesehen kann man die Frage auch umdrehen: Warum sollte er es nicht tun? Wenn seine Gesellschaft ihn dazu auffordert, weiter greifende Wirkungen seines Handelns zu reflektieren, wenn sie ihn dazu erzieht und wenn sie es ihm ermöglicht ${ }^{6}$, eine möglichst differenzierte Rationalität seiner Verantwortungen herauszubilden - und das nicht nur im Binnenverhältnis seines Hoheitsraumes, sondern auch in den ,außenpolitischen“ Bezügen -, dann wird er es voraussichtlich tun. Als kluger Regent seiner eigenen Welt wird er die zunächst zweckrational motivierte, aber dennoch deliberierende Rückfrage an seine Geschäftspartner stellen, ob eine schleichende physiologische Zerstörung der Kundschaft nicht langfristig geschäftsschädigende Gefahren wie Ächtung, Glaubwürdigkeitsverlust oder sogar krankheitsbedingten Zielgruppenschwund mit sich bringen könnte. Vielleicht wird er sich sogar als aktiver Gestalter einer Gesellschaft fühlen, deren Solidarität er durch solche Akte der kurzsichtigen Bereicherung in Gefahr sieht und in der Folge seine Geschäftspartner auffordern Frühstücksflocken herzustellen, die mit ihrer eigenen Kommunikation im Einklang stehen. Die deliberative Rückfrage wird zum vernunftbildenden Prinzip.

$5 \quad$ Etzioni (1994: 119ff.) stellt beispielsweise „,starke empirische Evidenzen gegen die Aussagen der Public Choice-Schule“ fest. Er verweist auf zahlreiche Experimente, in denen das ,pro-soziale“ Verhalten die Regel war. Er beschreibt unsere Gesellschaften als „,responsive communities“, als zur „Verantwortung fähige Gemeinschaften“ (ebd. 1994: 31).

6 Denn wir dürfen trotz aller Offensichtlichkeit der schädlichen Auswirkungen so hoher Zuckeranteile eines Kinderfrühstücks nicht die Möglichkeit außer Acht lassen, dass eine solche Produktkommunikation durch die ausführenden Akteure doch aus Unachtsamkeit, Unreflektiertheit und mangelnder Sensibilität heraus geschieht. Damit die schädlichen Auswirkungen auf ein bildungsfernes Milieu in diesem Beispiel bewusst werden, sollten also möglicherweise Ressourcen wie Zeit, Diskussion und Information zur Verfügung gestellt werden: Die Herausbildung einer Verantwortungsrationalität muss dem gehetzten Akteur ermöglicht werden. Er muss dazu motiviert werden. So wurden in der experimentellen Spieltheorie bei Dilemma-Situationen durch Dawes, van den Kragt und Orbell frappierende Abweichungen (teils mehr als 100\% Zuwachs) zugunsten eines sozialen Verhaltens erzielt, wenn die Spieler sich vorher gegenseitig kurz kennenlernten (vgl. Kesting 1999: 17). Zuwächse in der sozialen Kooperation und der Übernahme einer anonymen Verantwortung lassen sich in einem bestimmten Maß vermutlich auch auf ein abstraktes Kennenlernen und Wissen um die Situation und Existenz anderer durch Diskurse oder Berichte übertragen. 
Unsere derzeitige Realität sieht allerdings völlig anders aus. Der Mensch erlebt in seiner arbeitsteiligen Welt eine Vielzahl von „Dissoziationen“ (Hirschman 1984: 147)7, die ihn von der Ausübung seiner hoheitlichen Befugnisse abhalten. Debatin und Funiok beschreiben für die Medienwelt, wie es zu einem „Auseinandertreten von Entscheidungs-, Handlungs- und Verantwortungssubjekten“" (Debatin/Funiok 2003: 9) kommt. Diese Beobachtung gilt stellvertretend für jede hoch arbeitsteilige Branche. Da die Subjekte sich in ihrem wirtschaftlichen Handlungsprozess nicht auf ihre vollständige Weltwahrnehmung und Einschätzung beziehen können, sondern lediglich effizienzorientierte Teilperspektiven der jeweils zugewiesenen Rolle einnehmen, kommt es in der Summe zu einer Art Systemblindheit der Organisationen. Eine vollständige Verantwortung wird ausschließlich den vermeintlich Regierenden in den höheren Etagen zugewiesen. ${ }^{8}$ Aber auch diese übernehmen Verantwortung im Wesentlichen nur innerhalb der Grenzen der ökonomischen Sachlogik. Im sozialen Schadensfall wird von dort die Verantwortung dann auch gerne wieder zurückverwiesen, da man beispielsweise mit der problematischen Herstellung oder dem unmoralischen Vertrieb ${ }^{9}$ des Produktes im Einzelnen ja gar nichts zu tun hatte. Eine Rationalität der Verantwortung, eine Kunst des Regierens, entwickelt sich in diesen Verhältnissen nicht; riesige Verantwortungsdeltas öffnen sich deswegen, die für das Unternehmen, besonders aber für Gesellschaft, Umwelt und Individuum höchst schädlich sind.

In manchen Unternehmen ist diese Problematik auch schon erkannt worden. Rafael Capurro schreibt in den Geschäftsgrundsätzen von IBM:

„Das Prinzip der Vermeidung eines Interessenkonfliktes ist offensichtlich unmoralisch. Es müsste heißen: Bleiben Sie wach für Interessenkonflikte und meiden Sie diese nicht, sondern stellen Sie stets Ihre moralische Verantwortung über Ihre Rollenverantwortung!“ (Capurro 2003: 180)

Allerdings ist die Wertschätzung solcher moralischer Interventionen alles andere als üblich. Die Rollenverantwortung zugunsten einer moralischen Verantwortung zu negieren, ist für viele Akteure ein Ding der Unmöglichkeit, wenn sie nicht bereit sind, härteste soziale Konsequenzen dafür in Kauf zu nehmen. Viele diffundieren zwischen den beiden Elektroden des Schreckens, wie ich die subjektive Realität des modernen

$7 \quad$ Hirschman (1984: 147): „Die Abspaltung des Privaten vom Öffentlichen ist ein Merkmal und ein Problem, das geradezu wie eine Plage auf der modernen Gesellschaft liegt, aber sie ist nur eine von mehreren solcher Differenzierungen. Sie ähnelt in vielem der zuerst von Freud konstatierten Dissoziation von Arbeit und Liebe (...)."

$8 \quad$ Heinz K. Stahl beschreibt im Bezug auf die Deutungshoheit in Unternehmen einen vergleichbaren Systemprozess. Die Chefs sind die oberste „Deutungsinstanz“, die eine „Konstruktion von Wirklichkeit“"zu liefern haben (Hinterhuber/Stahl 2001: 12). Ähnlich verhält es sich mit einer Rationalität der Verantwortung. Frei nach dem Motto „Wenn wir ein solches Produkt in dieser oder jener Weise produzieren, dann wird das schon seine von oben verordnete Richtigkeit haben“, kann der jeweilige Akteur ethisch unreflektiert ausschließlich seiner zugewiesenen Rollenverantwortung gerecht werden.

9 Bei seinem Rücktritt als Aufsichtsratschef von Siemens wird Heinrich v. Pierer von der Süddeutschen Zeitung (Artikel vom 20.04.2007,,Rücktritt nach neuen Vorwürfen“) aus seinem Abschiedsbrief zitiert: „Eine persönliche Verantwortlichkeit mit Blick auf die laufenden Ermittlungen war nicht Grundlage meiner Entscheidung." 
Arbeitslebens beschreiben möchte. Auf der einen Seite droht Hartz IV mit all seinen (un)sozialen Implikationen und auf der anderen Seite der Auftrag mit seinen oft gesellschafts- oder umweltschädlichen Einforderungen. Der moderne Akteur ist gezwungen, sich für seinen Lebensunterhalt vollständig der Logik des jeweiligen Auftrages zu unterwerfen. Gerade im Bereich der Unternehmens- und Produktkommunikation, zu dem das Frühstücksflocken-Beispiel zählt, hat sich ein kreatives „Prekariat" (Stahl/Steyrer 2007: 65) ${ }^{10}$ in vollständiger Abhängigkeit von den großen Auftraggebern herausgebildet. Es hangelt sich von Auftragsvergabe zu Auftragsvergabe und erfüllt ausschließlich seine vom Unternehmen zugewiesene Rollenverantwortung, getrieben von seiner prekären wirtschaftlichen Situation und im Gehorsam gegenüber der in Briefings eingeforderten ökonomischen Effizienz; eine sensible moralische Reflexion findet vor dem Hintergrund der sozialen Bedrohungen kaum statt. Die Peristaltik des Systems scheidet Elemente mit zu hoher moralischer Sensibilität ${ }^{11}$ durch wiederholten Arbeitsplatzverlust oder Nichtbeauftragung konsequent aus. Durch diese Option verhalten sich die Akteure kognitiv dissonant und erklären das eigene Handeln für gut und richtig oder zumindest für unerheblich - ungeachtet ruinöser gesellschaftlicher und/oder ökologischer Auswirkungen.

Durch massive Umbrüche in Umwelt und Gesellschaft und zunehmend bedrohliche Zukunftsszenarien lässt sich derzeit allerdings eine schwelende Veränderung in der Haltung vieler Akteure beobachten. Die Offensichtlichkeit der ökologischen und sozialen Zerstörungen beginnt deren Verdrängungsmöglichkeit zunehmend zu übersteigen. Eine diffuse Scham greift um sich. Norbert Elias beschreibt, wie die „Schamschwelle“ im Zivilisationsprozess „in Schüben“ vorrückt und sich kongruent zu einer wachsenden „Vor- und Langsicht“ (vgl. Elias 1997: 410) entwickelt. Parallel zur zunehmenden Komplexität unserer Welt muss sich gerade in unserer bedrohten Zeit auch die Rationalität, also die „Vor- und Langsicht" der handelnden Akteure weiterentwickeln, um irreversible Zivilisationsschäden ${ }^{12}$ zu vermeiden. Heute verändern sich die Makrosysteme in einer beispiellosen Geschwindigkeit und der industrielle Produktionsprozess erzeugt dabei permanent neuartige globale Bedrohungen. Die zur Nationalstaatlichkeit verzwergte Rahmenordnung hat sich als zu unwirksam, grobmaschig und träge erwiesen, um die destruktiven Auswirkungen der gewaltigen globalen Produktionsmaschinerie nachhaltig zu regulieren. Die einzelnen Menschen aber empören sich zunehmend in Anbetracht der sich abzeichnenden Zerstörung der ökologischen

10 Stahl/Steyrer (2007: 65) zitieren einen Freelancer-Grafiker: „Die Proletarier - von den Landlosen im alten Rom bis zur ausgebeuteten Arbeiterschaft der industriellen Revolution - hatten als Besitz nur ihre Nachkommen (lat. proles); die Mitglieder des modernen Prekariats (Servio Bologna) haben nicht einmal diese."

Alford (2002: 1) zitiert einen Whistleblower: "If I had to do it again, I wouldn't blow the whistle for a million dollars. It ruined my life." Whistleblower sind das Extrembeispiel in einer Extremsituation für viele, die moralisch bzw. ethisch resignieren, weil sie ahnen, dass sie einer zerstörerischen Maschinerie dienen, sich aber außerstande sehen, innerhalb ihrer oder durch ihre Tätigkeit etwas zu verändern.

12 Ott (2007: 9): „Die Menschheit befindet sich an einem Scheideweg. Unser Handeln in den nächsten 10 bis 15 Jahren wird die Lebensbedingungen unserer Kinder, Enkel und deren Nachkommen entscheidend beeinflussen." 
und sozialen Lebensgrundlagen ihrer Kinder. Diejenigen, die sich aufgrund ihrer wirtschaftlichen Abhängigkeit nicht artikulieren dürfen, verspüren eine unterirdische Scham. Wir beobachten derzeit, wie sich das fehlende Regulativ unseres Systems im Schamempfinden der Subjekte gegenüber den nachfolgenden Generationen und dem eigenen Treiben niederschlägt. Hilge Landwehr meint: „Schamsituationen bieten die Chance, dass durch den plötzlichen Perspektivwechsel Normen artikulierbar werden, an denen man sich bis dahin ganz selbstverständlich und unreflektiert orientiert hat" (Landwehr 1999: 82). Auch wenn sich unsere heutige Scham aus der Geburt neuer Normen in einer neuen Weltsituation und nicht aus bereits bestehenden ableitet, ist das mögliche Zur-Sprache-Finden des von der ökonomischen Handlungstheorie zum Schweigen ${ }^{13}$ verurteilten homo oeconomicus eine riesige Chance. Die damit mögliche Rückverortung von Moral und Verantwortungsrationalität in den Verfügungsbereich eines jeden Einzelnen ist das fehlende Korrektiv für ein System, in dem sich bislang schweigend verantwortungsbefreite Erfüllungsgehilfen wechselseitig und ethisch bestens legitimiert die Steigbügel zur ökologischen und sozialen Zerstörung hielten. Die deliberative Rückfrage, die dieser aufkeimenden Scham der Gestalter als grundlegendes Kommunikationsprinzip der Wirtschaftssubjekte folgen kann, wird dementgegen eine Phänomenologie des Verantwortens hervorbringen. Die möglichen Auswirkungen des eigenen Handelns kommen zur Sprache. Es werden Zusammenhänge offenbar, die heute noch im Verborgenen wirken und für die wir bisher noch keine Begriffe entwickelt haben. Wenn wir die Auswirkungen unseres Handelns kommunikativ zulassen, also in deliberativen Diskursen zur Sprache bringen und auf den ,ontologischen Vorhang der Kommunikation“ (Bittencourt et al. 2004: 33) projizieren, können wir sie vermeiden bzw. gezielt anstreben.

Nur erleben wir leider gleichzeitig eine dramatische Verwahrlosung des sprachlichen Dispositivs. Dieselben Akteure, die in der Welt des Wirtschaftens über deliberative Diskurse längst eine Rationalität der Verantwortung entwickeln müssten, organisieren stattdessen fraglos und in der Summe mit Milliardenetats eine gigantische Maschinerie der Unaufrichtigkeit. Das Heer der Getriebenen erschafft über die werbefinanzierten Medien für unsere Demokratie und letztlich für sich selbst eine Konstruktion von Wirklichkeit, die größtenteils ökonomischen Zwecken unterworfen ist. Die inzwischen allgegenwärtige Werbung arbeitet dabei mit einem Zusammenspiel aus Überhöhung und Weglassung. Im Verhältnis zu einem Informationsanspruch des Bürgers kann man in sehr vielen Fällen von gezielter Desinformation sprechen. Im journalistischen Bereich existiert außerhalb des öffentlich-rechtlichen Raumes kaum eine Redaktion, die nicht in direkter Abhängigkeit zu den Werbegeldern der großen Unternehmen steht. Themen, Artikel, Beilagen, ja ganze Zeitschriften und Fernsehsender werden ausschließlich nach Relevanz für die werbetreibende Wirtschaft entworfen oder stehen auch im Falle hochwertiger Organe unter dem subkutanen Einfluss eines latent drohenden Anzeigenentzuges.

Vor Derrida/Stiegler hat 1956 bereits Günter Anders gewarnt, dass unsere Gesellschaften dabei sind, in ein ,postliterarisches Analphabetentum“ (Anders 1985: 3) 
abzugleiten. ${ }^{14}$ Viele verstehen weder die massenmedialen Herstellungsprozesse und ihre intentiösen Montage- und Auswahltechniken, noch identifizieren sie die hinter den Sendern und Verlagen wirkenden „neufeudalen Machtgruppen“15 und deren Interessenlagen; aber sie sind trotzdem der manipulativen Absicht des medialen Erzeugnisses ausgesetzt. Sie übernehmen Duktus, Gestus und die inhärente Moral der ökonomisch produzierten Kulturerzeugnisse. Die institutionalisierte Unaufrichtigkeit des medialen Produktionsprozesses, bei dem Profis verschiedenster Herkunft gegen ein Salär Teilausschnitte einer Scheinwirklichkeit produzieren, sedimentiert in Sprache und Haltung der gesamten Gesellschaft. Schönheitsoperationen nehmen drastisch zu und eine ganze Generation wird derzeit unter dem ästhetizistisch verzerrten Schönheitsideal von „Germany’s Next Topmodel“ individuiert. Die von Günter Anders diagnostizierte prometheische Scham, ,geworden, statt gemacht“ (Anders 1985: 24) zu sein, wird im Angesicht gecasteter, geschminkter, retuschierter und allgegenwärtig projizierter Gespenster zum allgemeinen Lebensgefühl. Spiegelgleich zur beschämten Verzwergung der Rezipienten regt sich allerdings auf der Seite der Produzenten inzwischen wiederum die diffuse Scham der Gestalter. Sie ${ }^{16}$ beginnen zu ahnen, was für einen unentrinnbaren Sumpf aus unerreichbaren Wünschen und Projektionen sie ihren Kindern da bereiten - unter wirtschaftlichem Zwang bereiten müssen. Sie sehen sich gezwungen, mit ihrem Tun jene zerstörerischen Lüste zu befeuern, welche die industrielle Nachfrage anheizen. Gleichzeitig werden aber die wirklichen Abgründe der Gegenwart durch diesen ungeheuerlichen „Medienmüll“ (Schneider 2003: 33) überlagert. Was wir daraus ernten, ist eine gefährliche Deformation des öffentlichen Bewusstseins. Wo wackere Demokraten sich im Diskurs um die bedrohte Zukunft gegenseitig mit differenzierten Konzepten überbieten müssten, beobachten wir ein medial sediertes Volk, das im Totalkonsum dem ökologisch-sozialen Niedergang entgegendämmert, unfähig, den demokratischen Prozess zu vollziehen. Das von

14 Derrida/Stiegler (2006: 71-74): „Die Technik der alphabetischen Schrift und die weitverbreitete Fähigkeit, sie zu verwenden, waren die Bedingung für die Konstitution von Staatsbürgerschaft. Die Teilhabe an dieser Fähigkeit hat sich fortwährend verbreitert; Jules Ferry bezeichnet den Punkt ihrer modernen Vollendung, doch sie begann schon im antiken Griechenland. Von den audiovisuellen Techniken unterscheidet sie sich vor allem darin, dass man nicht Leser von Büchern sein kann, ohne auf die eine oder andere Weise potentiell Schriftsteller zu sein: Es ist kaum vorstellbar, dass der Adressat des Buches es wirklich lesen könnte, ohne schreibkundig zu sein. Vielleicht wird er niemals schreiben, doch er liest, seitdem er mit dem Erwerb der Lesefähigkeit die Möglichkeit zu schreiben hat. Vor allem aus technischen Gründen erlauben es die audiovisuellen Medien und der Computer hingegen, dass ein Empfänger keinerlei Kompetenz mehr hinsichtlich der Genese - der Produktion dessen, was er empfängt - benötigt.“

15 Eucken (1990: 234) wird von Ulrich zitiert: „Die Politik des Staates sollte darauf gerichtet sein, wirtschaftliche Machtgruppen aufzulösen und ihre Funktion zu begrenzen. Jede Festigung der Machtgruppen verstärkt die neufeudale Autoritätsminderung des Staates" (Ulrich 2001: 351).

Nachdem ich selbst seit vielen Jahren in der Fernseh-/Werbebranche tätig bin, müsste ich gerechterweise von „uns“ und „wir“ sprechen, bleibe aber aus formalen Gründen in der dritten Person. Ich habe in vielen Gesprächen mit Gestaltern der Massenmedien aus unterschiedlichsten Bereichen festgestellt, dass es kaum jemanden gibt, der diese diffuse Scham nicht kennt und sich nicht dringend eine qualitative Veränderung des Mediensystems wie auch eine moralisch/ethische Qualifizierung des Wirtschaftssystems wünscht. Viele haben allerdings resigniert, angesichts der gewaltigen Mechanismen, die hier am Werk sind. 
Habermas gewünschte „Warnsystem mit (...) gesellschaftsweit wirksamen Sensoren“ (Habermas 1998: 435) ist durch die ökonomische Anästhesierung des Mediensystems und seines Publikums wirkungslos geworden. Es warnt nicht, solange kein ökonomisches Interesse hinter der Warnung wirkt ${ }^{17}$. Der Markt hat sich über die „Logik des Kommerz" (Bourdieu 1998), von der das Massenkommunikationssystem durchwirkt ist, vom wissenschaffenden „Entdeckungsverfahren“ (vgl. Hayek 1969) (vgl. zur „Offenbarungstheorie des Marktes“ Ulrich 2001: 173) zu einem gigantischen Verschleierungsmechanismus verwandelt, der seinem Publikum mit einer beschämenden „Ikonomanie“ des Banalen die „Augen stopft“ (Anders 1985: 3). Es bestätigt sich einmal mehr McLuhans (1992) Analyse bezüglich des affirmativen Verhältnisses der Massenmedien zu den bestehenden Herrschaftsstrukturen - in unserer Gesellschaft allerdings nicht mehr der politischen Herrschaftstrukturen, sondern der ökonomischen. ${ }^{18}$

All das sind im Verhältnis zum unterstellten Hoheitsraum des einzelnen Wirtschaftssubjektes „außenpolitische“ Probleme. Die philosophiegeschichtliche Analogie vom Schiffsführer, dessen Verantwortung sich auf Ladung, Schiff und Mannschaft erstreckt, erfasst die gerade beschriebenen „außenpolitischen“ Zusammenhänge auf den ersten Blick nicht. Um die weitreichenden Verantwortungen des heutigen Wirtschaftens metaphorisch zu aktualisieren, müsste man den Schiffsvergleich Foucaults auf einen jener Trawler beziehen, die Schleppnetze mit tonnenschweren Gewichten über den Meeresboden ziehen und diesen damit derart „nachhaltig“ vernichten, dass er in seiner Entwicklung in ein erdzeitliches Frühstadium zurückfällt. Der Kapitän eines solchen Schiffes nimmt vermutlich seine klassischen Verantwortungen nach herkömmlicher Denkart korrekt wahr - bringt er doch Schiff, Ladung und Mannschaft heil und rechtzeitig in den heimatlichen Hafen. Aber reicht dieses Denken noch aus? Mit der Zerstörung der Fanggründe vernichtet er sowohl seine eigene Ernährungsgrundlage als auch die seiner Mannschaft. Vollzieht er damit einen Akt des Regierens oder eher der ökonomischen Zwangsverwaltung? Ist er ein Regierender oder eher Erfüllungsgehilfe höherer Autoritäten? Wie verhält es sich mit dem CEO der Fangflotte? Auch er vernichtet mit einer solchen Politik die Lebensgrundlage seiner eigenen Firma, ja seiner gesamten Branche und, wenn man manch düsterem Szenario

Ich habe Gespräche mit Eckhard Kuhla geführt, der sich mit seiner Gruppe moin seit Jahren dafür einsetzt, wider das bedrohlich heranrückende Peak-Oil-Phänomen zu einem nationalen Aktionsplan und letztlich endlich zu einer Energiewende zu kommen. Er hat mir geschildert, wie ihm in Gesprächen mit Privatsendern (Kontext Wissensformate) eine ganze Sendereihe zu dem ,interessanten Thema" angeboten wurde, sofern er einen Sponsor mitbringt, der das Format finanziert. Man sieht an diesem Beispiel: Das Warnsystem Öffentlichkeit warnt nicht mehr, solange kein ökonomisches Interesse dahinter wirkt!

18 Es wäre interessant zu untersuchen, inwieweit zeitgleich mit Aufkommen des Privatfernsehens in Deutschland nachweislich eine Machtverschiebung vom politischen zum ökonomischen System stattgefunden hat und McLuhans (1992) Analyse dahingehend differenziert werden kann, dass sich mit dem Erscheinen eines neuen Massenmediums die Machtverhältnisse zugunsten der jeweils dahinter wirkenden Entscheidungs- und Gestaltungsgruppen (hier die Wirtschaft) verschieben. In der Folge stellt sich die Frage, ob mit dem Aufkommen des Internet bürgerschaftliche Gruppen (NGOs, NPOs) einen gesellschaftlichen Machtzuwachs erfahren haben oder noch erfahren werden. Eine solche Untersuchung würde möglicherweise in einer Hypothese zum idealtypischen medialen Dispositiv für die deliberative Demokratie münden. 
Glauben schenken will, sogar der Menschheit. Regiert er oder ist er wiederum nur Erfüllungsgehilfe höherer Autoritäten, beispielsweise der Shareholder? Sind diese oft anonymen Shareholder dann diejenigen, von denen man eine Kunst des Regierens einfordern könnte? Nun, vermutlich eher nicht. Wir müssen feststellen, dass in diesem Beispiel gar niemand regiert. Es handelt sich um unregierte Flotten, die marodierend über die Ozeane ziehen, im Dienste einer Apokalypse, für die niemand die Verantwortung trägt und die von keiner maritimen Rahmenordnung gebremst werden kann.

Die herkömmlichen Institutionen versagen ${ }^{19}$ bei der Bändigung des ökonomischen Raubtieres, das gerade dabei ist, unsere Zukunft zu negieren. Es gibt allerdings eine Institution, die hier Rettung verspricht und bisher vernachlässigt wurde: der Mensch. Und zwar der vollständige, nicht dissoziierte Mensch, im vollen Bewusstsein seiner Verantwortung, als Regierender seines eigenen Lebens. Als Gestalter, der im deliberativen Diskurs eine Verantwortungsrationalität herausbildet und seine Handlungen danach richtet. Eine solche simple und zutiefst menschliche (Wirtschafts-)Anthropologie braucht Flächen, Räume und Orte, an denen sie sich kraftvoll entfalten kann. Die aufkeimende Scham der Gestalter ist möglicherweise der Performancetreiber, um in eine Bewegung zu münden, die ein neues, nachhaltiges und endlich auch würdevolles Wirtschaften hervorbringt; nennen wir es ruhig eine Revolution, die von all jenen betrieben wird, die Zukunft aktiv gestalten wollen, ungeachtet ihrer beruflichen Herkunft.

Für die Wirtschaftsethik sehe ich die Chance, die diskursive Selbstorganisation einer hoch entwickelten Verantwortungsrationalität sämtlicher Akteure in all ihren Potenzialen, Blockaden und Systemzusammenhängen zu reflektieren und durch intelligente normative Vorgaben zu dynamisieren. Die künstlichen Dissoziationen des Menschen (vgl. oben) und seiner Handlungen sollten dabei ebenso überwunden werden wie die Trennlinien der verschiedenen ethischen Sujets. Wer heute Wirtschaftsethik betreibt, wird nicht mehr umhin kommen, auch Medienethiker zu sein, wenn er nicht massive Aus- und Rückwirkungen seines Reflexionsbereiches außer Acht lassen will. In den Medien, den Unternehmen und der Gesellschaft gibt es gleichermaßen ein gefährliches Diskursdefizit, sowohl was die Quantität als auch die Qualität und die grundlegende Kompetenz angeht. Die wirksame Initiierung und die nachhaltige Qualifizierung von Diskursen sind zwei der dringlichsten Aufgaben unserer Zeit, und spätestens an dieser Stelle werden Medien- und Wirtschaftsethiker einmal mehr zu Staatsphilosophen und umgekehrt.

Ich halte es für das wichtigste Potenzial des Begriffes corporate governance, die Unternehmensführung in ihrer Verantwortungsrationalität als fraktale Spiegelung eines staatlichen Regierungshandelns zu reflektieren und in einer konsequenten Folgedis-

Etzioni (1994: 329): „die Ansicht von der automatisch und kollektiv zur Verfügung gestellten Rationalität, eine Rationalität, die es den Individuen erspart überlegen zu müssen, und sie doch rational handeln läßt, wird von den Fakten kaum untermauert und ist kaum haltbar." Ich beziehe diese von Etzioni diagnostizierte Untauglichkeit von Regeln zur Implementierung von Rationalität in die Gesellschaft auch auf die Implementierung einer Verantwortungsrationalität durch eine reine Institutionenethik. Die Institutionen sollten sich nicht darauf beschränken, Regeln aufzustellen, sondern vielmehr durch ihre fördernden und einfordernden Möglichkeiten eine diskursive Rationalität in und zwischen den Subjekten induzieren; eine Rationalität, die sich über einen verstärkt partizipativen Prozess dann auch wieder in den Institutionen und Regeln abbildet. 
kussion sämtliche Implikationen von Regierungskunst, bis hin zur Konstituierung der Regierung oder der demokratischen Souveränitätsverortung, auch im Bereich der Unternehmen und Organisationen mit den Errungenschaften der demokratischen Moderne abzugleichen. Es wäre wünschenswert, dass die CSR-Manager eine so weitreichende Reflexion des Governance-Denkens auch in den beschriebenen ,„außenpolitischen" Bezügen in die Unternehmen einbringen und mit ihren höchsten Repräsentanten als Machtpromotoren eines transformativen Prozesses auf normativer Managementebene angesiedelt werden. Die Ermunterung des Stakeholders zur verantwortungsrationalen Deliberation kann nur auf dieser Ebene als Unternehmensziel verankert und in der Folge mit den notwendigen Ressourcen ausgestattet werden. Institutionelle ethische Vorgaben auf Meso- und Makroebene werden damit keineswegs überflüssig, sondern vielmehr Resonanzobjekt und Stimulus für die wichtigste aller Institutionen: den Menschen und seine Vernunftbildung.

Freier Markt und Demokratie werden von vielen Enthusiasten des Neoliberalismus als Zwillingspaar ${ }^{20}$ gefeiert. Ein Problem an dieser Spaltung des gesellschaftlichen Handlungsraumes ist, dass eine diskursiv-demokratische Rationalität in der Konstruktion des freien Marktes keinen Platz findet, sondern in eine demokratische Anderswelt verwiesen wird. Das menschliche Handeln und die menschliche Psyche werden durch diese künstliche Teilung gespalten. Der Mensch soll als Wirtschaftsakteur im Wettbewerb egoorientiert Profite ${ }^{21}$ machen, gleichzeitig aber als Demokrat, Wähler und Souverän sozioorientiert eine Rahmenordnung mitkonstruieren, die ihn in diesem Streben zügelt. Statt ihm dieses handlungs- und demokratietheoretisch konfligierende Jekyll-Hyde-Dilemma zuzumuten, sollte man ihn einladen, sein Handeln schon beim Handeln ethisch ${ }^{22}$ zu reflektieren und dabei gemeinsam mit anderen ein Ethos herauszubilden. Ethik, Schulerziehung und Medien sollten dazu gemeinsam und nachhaltig endlich den Aufbruch in die deliberative Moderne wagen. Die heute keimende Scham $\operatorname{der}$ Gestalter ist möglicherweise auch in diesen Subsystemen einmal mehr der Impulsgeber für diesen dringend notwendigen Prozess und damit hoffentlich Korrektiv unseres heißlaufenden Systems.

Nun wird man sich fragen, was der Kapitän des apokalyptischen Trawlers tun soll, wenn er sich vom gezwungenen Zwangsverwalter zum Regierenden verwandeln will. Ich kann diese Frage nicht beantworten, denn ich kenne die Lebenssituation dieser hypothetischen Figur natürlich nicht. Aber ich vermute, dass es für jede Aufgabe eine Logik gibt, wie man einem verantwortungsrationalen Handeln näher kommt. Manchmal ist es die Negation der Aufgabe, manchmal ihre Verwandlung und manchmal wohl auch ihre Erfüllung. Als die Firma Hertie Anfang 2008 bekannt gab, dass sie eine

20 Barber (1996: 18, 19): „Zu behaupten, Demokratie und freier Markt seien Zwillinge ist ein Allgemeinplatz der Staatsoberhäupter geworden. "Barber beschreibt, wie Clinton 1994 bei seinen Besuchen Osteuropas ,gebetsmühlenartig“ von den „democratic markets“ spricht.

21 Die wenigsten machen Profite, sondern hetzen vielmehr ihrem Lebensunterhalt hinterher.

22 Ich meine damit, dass man auch dem normalen Menschen durchaus zutrauen darf, auf einer normativen Reflexionsebene die gängige handlungsleitende Moral zu reflektieren und mit zu entwickeln. Die Entmündigung des Subjekts sollte seiner schrittweisen Befähigung weichen und diese beginnt mit dem grundsätzlichen (normativen) Ver- und Zutrauen durch die Vordenker. 
neue Werbeagentur sucht, habe ich vorgeschlagen, das Werbebudget in den kommenden Jahren konsequent zu halbieren und die frei werdenden Gelder dafür zu verwenden, eine Redaktion aufzubauen, die nach und nach jedes Produkt, das in den Kaufhäusern verkauft wird, bis zu seinen absoluten Anfängen recherchiert. Die Ergebnisse sollten den Verbrauchern im Internet zur Verfügung gestellt werden. Mit der Zeit würde sich daraus ein Hertie-Gütesiegel ableiten, auf das sich in der Folge auch die Hersteller einstellen. Hertie würde damit zum Qualitätsdienstleister der Verbraucher und zum Qualitätsimpulsgeber der Hersteller; gegenüber den Mitbewerbern würde die Marke eine Alleinstellung erhalten und gleichzeitig zum angesehenen Vorreiter des sich in Richtung Glaubwürdigkeit drehenden Marktes. Der desinformative Grundcharakter von Werbung würde sich zu einer vollständigen Information verwandeln ${ }^{23}$ und damit vielleicht sogar zum Trendsetter für eine verantwortungsvolle werbliche Selbstrepräsentation eines großen Unternehmens. Mit den sonst mehr oder weniger austauschbaren Werbespots würde ein Stück Medienmüll verschwinden und mehr Relevanz ins öffentliche Bewusstsein zurückkehren. Es würde ein Werbejob entstehen, für den sich niemand zu schämen braucht, sofern die Arbeit gewissenhaft gemacht wird ja vielleicht würde sich die diffuse Scham der Werbegestalter sogar in Stolz ${ }^{24}$ verwandeln, einen wichtigen Beitrag zur Transformation unserer Gesellschaft zu leisten. Ich weiß nicht, inwieweit ein solcher Kommunikationsvorgang bereits als verantwortungsrational zu bezeichnen ist, weil sich hier noch keine Normen gebildet haben und es auch keine wahrnehmbaren Flächen für die dazu notwendigen Diskurse gibt. Jedenfalls stellt sich dieser Vorschlag einem hypothetischen Diskurs darüber und versucht dabei, ein neues Denken anzuregen. Ein neues Denken, eine neue Rationalität der Verantwortung bilden jene Interventionen, welche Markt, Subjekt, Gesellschaft und Umwelt dringend brauchen. Dieses neue Denken kann sich nur in den Diskursen aller Wirtschaftssubjekte entwickeln und entfalten. Wir sollten unser Handeln mit einer immerwährenden und allgegenwärtigen Konferenz begleiten, in der sich diese Rationalität der Verantwortung herausbildet, um so zu einer wirklichen governance zu kommen und die massiven Gefahren für unsere Zukunft zu überwinden.

\section{Literaturverzeichnis}

Alford, F. C. (2002): Whistleblowers, Broken Lives and Organisational Power, Cornell: Cornell University Press.

Anders, G. (1956/1985): Die Antiquiertheit des Menschen. Über die Seele im Zeitalter der zweiten industriellen Revolution, Band 1, München: C. H. Beck'sche Verlagsbuchhandlung.

Barber, B. R. (1996): Coca Cola und Heiliger Krieg. Wie Kapitalismus und Fundamentalismus Demokratie und Freiheit abschaffen, Bern u. a.: Scherz Verlag.

Bittencourt, I./ Borner, J./ Heiser, A. (2004) (Hrsg.): Nachhaltigkeit in 50 Sekunden, München: Oekom Verlag.

Funiok (1981: 406): „Wagner (1978) möchte die Werbung sogar zu einer ,kompletten Darstellung des Wissens und Urteilens über das angebotene bzw. produzierte Produkt (a. a. O) verpflichten' - eine Erwartung, die im Blick auf die Realität der heutigen Werbung sehr hoch angesetzt ist.“

Auch hier bin ich natürlich wieder selbst in gleichem Maße betroffen. 
Bourdieu, P. (1998): Über das Fernsehen, Frankfurt a. M.: Edition Suhrkamp.

Capurro, R. (2003): Ethik im Netz, in: Funiok, R. (Hrsg.): Medienethik - Verantwortung in der Mediengesellschaft, Stuttgart: Verlag Kohlammer.

Debatin, B./ Funiok, R. (Hrsg.) (2003): Kommunikations- und Medienethik, Konstanz: UVK Verlagsgesellschaft mbH.

Derrida, J./ Stiegler, B. (2006): Echographien: Fernsehgespräche, Wien: Passagen Verlag.

Elias, N. (1997): Über den Prozess der Zivilisation. Soziogenetische und phylogenetische Untersuchungen, Band 1: Wandlungen des Verhaltens in den weltlichen Schichten des Abendlandes, Frankfurt a. M.: Suhrkamp.

Etzioni, A. (1994): Jenseits des Egoismus-Prinzips. Ein neues Bild von Wirtschaft und Gesellschaft, Stuttgart: Schäffer-Poeschel Verlag.

Eucken, W. (1952/1990): Grundsätze der Wirtschaftspolitik. 6. durchges. Aufl., hrsg. v. E. Eucken und K. P. Hensel, Tübingen: J. C. B. Mohr.

Foucault, M. (2004): Geschichte der Gouvernementalität, Band 1: Sicherheit, Territorium, Bevölkerung, Frankfurt a. M.: Suhrkamp.

Funiok, R. (1981): Fernsehen lernen - eine Herausforderung an die Pädagogik: Theorie und Praxis d. Fernseherziehung, München: Minerva Publikationen.

Habermas, J. (1998): Faktizität und Geltung, Beiträge zur Diskurstheorie des Rechts und demokratischen Rechtsstaats, Frankfurt a. M.: Suhrkamp.

Hayek, F. A. v. (1969): Der Wettbewerb als Entdeckungsverfahren, in: Ders. (Hrsg.): Freiburger Studien, Tübingen, 249-264.

Hinterhuber H. H./ Stabl H. K. (Hrsg.) (2001): Fallen die Unternehmensgrenzen? Beiträge zur Außenorientierung der Unternehmensführung, Renningen: Expert Verlag.

Hirschman, A. O. (1984): Engagement und Enttäuschung, Über das Schwanken der Bürger zwischen Privatwohl und Gemeinwohl, Frankfurt a. M.: Suhrkamp.

Homann, K./ Blome-Drees, F. (1992): Wirtschafts- und Unternehmensethik, Stuttgart: UTB.

Homann, K. (1994): Ethik und Ökonomik, in: Ders. (Hrsg): Wirtschaftsethische Perspektiven I, Berlin: Duncker \& Humblot, 9-30.

Kesting, S. (1999): Diskurs und Macht. Ein Beitrag zur ökonomischen Handlungstheorie, Pfaffenweiler: Centaurus Verl. Ges.

Landwehr, H. (1999): Scham und Macht, Phänomenologische Untersuchung zur Sozialität eines Gefühls, Tübingen: J. C. B. Mohr.

McLuhan, M. (1968/1992): Die magischen Kanäle. Understanding Media, Düsseldorf: Econ Verlag.

Ott, H. E. (2007): Wege aus der Klimafalle: Neue Ziele, neue Allianzen, neue Technologien was eine zukünftige Klimapolitik leisten muss, München: Oekom Verlag.

Schneider, J. (2003): Der systematische Ort der Medienethik - Eine sprachphilosophische Perspektive, in: Debatin B./ Funiok R. (Hrsg.): Kommunikations- und Medienethik, Konstanz: UVK Verlagsgesellschaft $\mathrm{mbH}$.

Stabl, H. K./ Steyrer, J. (2007): Change Management im Wanderzirkus Unternehmen, in: OrganisationsEntwicklung - Zeitschrift für Unternehmensentwicklung und Change Management, 2007/Nr. 4, 65-72.

Ulrich, P. (1997/2001): Integrative Wirtschaftsethik, Grundlagen einer lebensdienlichen Ökonomie, Bern, Stuttgart, Wien: Haupt.

Wagner, H. (1978): Kommunikation und Gesellschaft, Teil I: Einführung in die Zeitungswissenschaft (=Soziale Kommunikation in Theorie und Praxis 1), München: Olzog. 